\title{
Reinforcement fibers in concrete envelopes of underground nuclear power stations
}

\author{
V. Doležel ${ }^{1} \&$ P. P. Procházka ${ }^{2}$ \\ ${ }^{1}$ University of Pardubice, Czech Republic \\ ${ }^{2}$ Assoc. of Czech Concrete Engineers \& CTU, Prague, Czech Republic
}

\begin{abstract}
Underground spaces offer large areas or volumes for establishment of underground nuclear power stations, underground halls, underground deposits of nuclear waste, and underground sewerage plants, etc. The roofing of such structures requires thick walled structures, in most cases being created from fiber reinforced concretes. Additionally, standard rebars serve as a bearing reinforcement while the fibers keep off moisture, chemical gas, vapor, which can cause damaging corrosion of the rebars of various kind. The fibers serve also as defense from influence of relaxation due to change of temperature. Since the structures of this kind are of length span, the construction of them demands special treatment during the soil covering of the roof of such structures, which are here considered pelted. Moreover, very important phenomenon, creep, should be involved in the calculation as the time for building up such robust structures requires long period, during which the creep in particular parts of the structure can influence the stress state in the whole structure. The starting idea is based on creation of lathwork supporting the whole structure. On this lathwork all parts of the concrete structure will be positioned in stages, which are prescribed with respect to successive loading and optimal bearing capacity in overall structure involving successive influence of creep.

In this paper, advantages of surface nuclear power stations, underground drilled power stations and pelted nuclear power stations are discussed and for the latter fiber reinforce concrete is discussed. Some results of tests of selected fibers aiming to application if structures of pelted power stations are presented. The influence of mechanical behavior, as well as the thermal and chemical effects is shown.
\end{abstract}

Keywords: underground power stations, pelted nuclear power stations, fiber reinforcement. 


\section{Introduction}

It seems likely that many of the world's states will soon begin to build many nuclear power stations; some for the first time and others after ending a longfrozen program. The reasons cited centre on climate change as it is true that, once operational, nuclear reactors are largely carbon-neutral. Furthermore, they have high energy density (very high power output from a very small space) and operate continuously over lengthy periods. All they do is provide a framework in which a controlled fission reaction within its uranium fuel heats up a primary coolant (circulating water or inert gas, contained under pressure). The super-hot coolant then heats water via a heat exchanger to raise steam to drive turbines to generate electric power. Renewables have low energy densities and operate intermittently regardless of the source of energy. At present, there is no viable way to store energy produced on a large enough scale to keep power available at all times; something we have come to expect. These factors, among others, make it inevitable that many new reactors will be built.

Given that reactors will be built whether we like it or not, how can we ensure that they are as safe as possible? Mention the word 'nuclear' to most people, and words like Chernobyl, Hiroshima, missiles, nuclear waste, Windscale and Three Mile Island trip into the mind. Nuclear power has not, over the years, had a good press. Yet it could easily be made much safer.

It is necessary top take into consideration nuclear power hazards. These are well known so we'll just briefly review them. The hazards all stem from the radiation produced by the primary heat-generating fission reaction, spent fuel rods, irradiated reactor assemblies, reprocessing (if any) and the resulting radionuclides which are created in the fissioning of uranium-235 atoms. The reactor is typically sealed in a primary containment vessel with radiation shielding surrounding it. These assemblies, in turn, are usually contained in a secondary reinforced concrete building which is designed to contain radiation products in the event of an accident in which the primary containment breaks down. There was no secondary containment at Chernobyl and the results of the partial meltdown that followed the doomed 'experiment' are now grim history.

\section{General strategy}

The underground structures suppose to be equipped with some important accessories. One of the most essential appears to be a defense from influence of chemically aggressive gas and such other matters, from moisture and vapor, suppressing volumetric changes during curing process of concrete envelope, and diminishing of impacts of relaxation due to change of temperature. Consequently, properly prepared fiber reinforced concrete has to be used for basic parts of the structure of power station.

Usage of existing software equipment for heating balance of power cycle for resolving the heat extraction and for resolving circulation of refrigerating media (thermo hydraulics of fuel zone, residual heat extraction to the atmosphere). 
Program systems based on FEM, BEM, and SHDM, which are contemporary available at University of Pardubice and Civil Engineering of the Czech Technical University will be used for comparative and combined problems. Program for calculation of average development of elastic constants of ground and terrestrial environment from deformation defined on physical model will be used for inverse analysis and interpretation.

The effective usage of mathematical models is limited by the fact that only in relatively very small field of set of tension Hooke's linear law governs in ground or terrestrial massive. A number of experiments to express physical non-linearity have been done in several last decades. However, this effort often dash against the basic ignorance of the physical law. Unless theoretically defined physical rule of law is not entirely speculative, it is necessary to result from the laboratory results obtained on specimens or fragments of the ground, eventually from metering in situ. In the first case the results have only limited relevance; in the second case some required tests are only very hardly viable and very capitalintensive.

Usage of the experimental method of physical simulation can entirely eliminate above-mentioned faults and problems with suitable strategy of experiments. Further this experimental method allows us progressive survey of transformation of particular substances to the limit of failure, what makes also combined simulation with usage of suitable formulated mathematical and physical models very attractive.

General process in combined analysis of mechanics of ground and soil problems involves:

1. Construction of geological profiles of the ground complex

2. Dividing planes will be marked out (bedding, foliation, fissures, cracks and dislocations), which notably influence resolving the task. Eventual control of the importance of particular dividing planes will be made on the physical models.

3. Relation between stresses, strains, deformation, speed of deformation and time by mechanical tests of homogenous or quasi-homogenous parts of the grounds are to be determined. The tests will be made in order to be able to appoint beginning and process of the grounds dilatation. It is necessary to choose appropriate development of the stresses before the failure of the system.

4. Mechanical characters of the dividing planes (deformation characteristics, cohesion, friction, dilation) and filling substances by measuring in the terrain are to be discovered.

5. A model on the physical similarity principles will be created to respond the ground complex.

6. Input parameters of the ground complex for mathematical solution of the problem will be prepared from the results of experiments on physical models. 


\section{Pullout problem of a steel FRC beam}

The pullout problem has frequently been solved in a problem of cracking of composite structures of several sorts. Since large span structures are considered here, locally large deformation pull out problem should be taken into account.

In this study of a lag model of the system concrete matrix - steel fiber is used. A finer mesh of the finite elements is necessary for the stability of an iterative process particularly in the neighborhood of expected nonlinear behavior on the contact (interface between fibers and matrix).

The debonding or "slipping" (jumps of responding points of the matrix and fibers boundaries in the tangential direction to the interface) can be caused due to several physical models. First, no tension tractions on the interface may occur. Also, some friction law (such as Coulomb law, Mohr-Coulomb law, shear bond strength are often introduced and debond parameters are computed. Since no fiber gripping force from the matrix is expected, the general Mohr-Coulomb friction law is restricted to the exclusion of shear stresses exceeding the shear strength in the tangential direction and the tensile stress exceeding the tension strength is also excluded along the interface between the fiber and the matrix.

The practical reason for applying large displacement theory shows the following Fig. 1. From this picture it is seen that the fibers are pulled out of the concrete and the behavior during the pulling process is much more complicated than when starting with small displacement theory. This is also the motivation of this study.

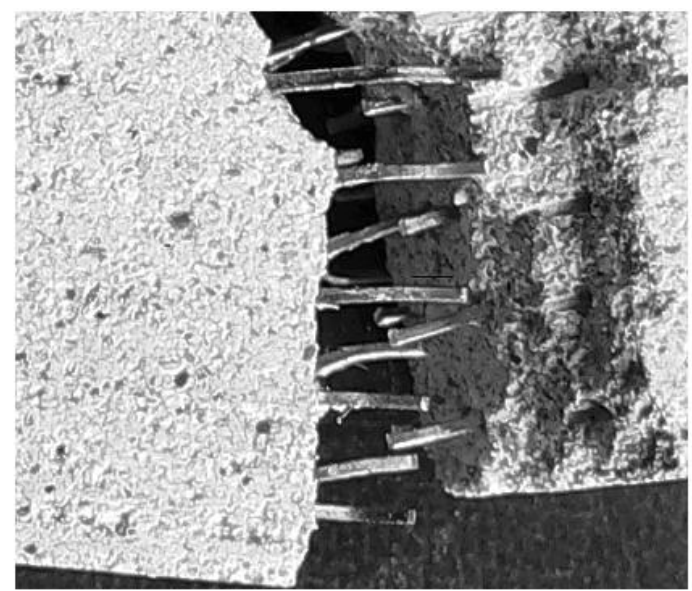

Figure 1: $\quad$ Detail of the cracked zone.

In the cracked zone it is seen that the fibers has been pulled out of the matrix in such a way that their shape is almost hold and the process of pull out is rather complicated then it was considered before. Curvilinear Dramix type fibers have been used. Similar situation can occur when straight fibers non-symmetrically 
positioned are applied, although the curvilinear are more sensitive of the way of loading.

Consider a contact problem of two bodies. The first body (fiber) occupies in undeformed state the domain $\Omega^{\prime}$ with the interfacial boundary $\Gamma^{\prime}$ (generally partly rectangular parallelepipeds with three connected parts, two are parallel and the second creates slope of the fiber) and the second body (concrete matrix) occupies the domain $\Omega^{\prime \prime}$ with the boundary $\Gamma^{\prime \prime}$, (parallelepiped), the fiber in which is embedded.

There is no external load considered but the pullout force at the face of the rectangular parallelepiped. The bodies are situated in Cartesian coordinate system $O x y z$; the axis $x$ is introduced in the radial direction of the lag. Generally, it will be assumed that the tension exceeding the tensile strength in the normal direction to the interface fiber-matrix $\Gamma$ is not admitted, so that both bodies may disconnect (they mutually debond) in certain region of the common boundary $\Gamma$, which is a part of $\Gamma^{\prime}$ and $\Gamma^{\prime \prime}$. The limit strength contact condition will be taken into account.

The load is considered in the following way. The pullout traction $F$ is applied at the face of the fiber in the axial direction, and prescribed by a starting constant value. Then the value is successively increased. There is symmetry about the vertical plane containing the horizontal axis $x$ regarding both geometry and loading, so that only one half of the unit cell may be solved.

Displacements are described by the vector function $\boldsymbol{u}=\{u, v, w\}$ of the variable $\boldsymbol{x}=\{x, y, z\}$. Denote $\Omega \equiv \Omega^{\prime} \cup \Omega^{\prime}{ }^{\prime}$. The restriction of any function to $\Omega^{\prime}$, or $\Omega$ " is denoted, respectively, by one prime or by two primes, e.g., $\boldsymbol{u} / \Omega^{\prime}=\boldsymbol{u}^{\prime}$ and $\boldsymbol{u} / \Omega$ " $=\boldsymbol{u} "$. On the boundary displacements and tractions are prescribed in such a manner that a periodicity is assumed at all sides of a "unit cell".

Denote the set of admissible displacements $\boldsymbol{u}$ on $\Omega$ satisfying the essential boundary conditions by $V$. The values and the first derivatives are quadratically integrable.

Consider Hooke's law in the form:

$$
\varepsilon_{i j}^{\prime}\left(u^{\prime}\right)=L_{i j k l}^{\prime} \sigma_{k l}^{\prime} \quad \varepsilon_{i j}^{\prime \prime}\left(u^{\prime \prime}\right)=L_{i j k l}^{\prime \prime} \sigma_{k l}^{\prime \prime}
$$

where $L^{\prime}$ and $L^{\prime \prime}$ are the material stiffness matrices of the fiber and the concrete, respectively. The overall stiffness matrices $\boldsymbol{K}$ relate nodal forces and nodal displacements as:

$$
\boldsymbol{K}^{\prime} \boldsymbol{u}^{\prime}=\boldsymbol{p}^{\prime} \quad \boldsymbol{K}^{\prime \prime} \boldsymbol{u}^{\prime \prime}=\boldsymbol{p}^{\prime \prime}
$$

Note that while the two primed stiffness matrix is regular one primed stiffness matrix is singular and will need certain necessary improvements after introducing the overall boundary conditions. This will be done in what follows.

Assuming "large deformation" theory, it may be satisfactory to formulate the essential boundary conditions on the contact boundary in nodal points of the elements (not necessary mutually adjacent) as follows (Signorini's conditions):

$$
[u]_{n}=u_{n}^{\prime}-u_{n}^{\prime \prime}<0 \quad \text { a.e. on } \Gamma
$$


Denote

$$
H \equiv\left\{\boldsymbol{u} \in V ;[u]_{n}<0 \quad \text { a.e. on } \Gamma\right\}
$$

The set $H$ is a cone of admissible nodal displacements with respect to the essential boundary and contact conditions, $V$ are displacements from the space of continuous functions.

Suppose that we disconnect both bodies under consideration, but keep the stress and deformation state in them "frozen". Then the vector of nodal contact tractions $\boldsymbol{p}=\left\{p_{x}, p_{z}, p_{y}\right\}$ must be introduced and their equilibrium (action and reaction law) says that:

$$
p=p^{\prime}+p^{\prime \prime}=0
$$

The shear bond strength condition with exclusion of tension read as:

$$
\begin{gathered}
p_{n}-p_{n}^{+} \leq 0, \quad\left[u_{n}\right]<0, \quad\left(p_{n}-p_{n}^{+}\right)\left[u_{n}\right]=0 \\
C=k\left(p_{n}-p_{n}^{+}\right) c,\left|p_{t}\right|-C \leq 0, \quad\left|\left[u_{t}\right]\right|>0, \quad\left(\left|p_{t}\right|-C\right)\left|\left[u_{t}\right]\right|=0,
\end{gathered}
$$

where $C$ is the cohesion (shear strength), $k$ is the modified Heaviside function being equal to one for negative arguments and zero otherwise, $\left[u_{n}\right]=u_{n}^{c}-u_{n}^{s}$, $\left[u_{t}\right]=u_{t}^{c}-u_{t}^{s}$, and $u_{n}^{c}, u_{n}^{s}$ are displacements normal to the steel - concrete boundary along the interface with respect to the concrete and the steel, respectively, and $u_{t}^{c}, u_{t}^{s}$ are tangential displacements along the interface with respect to the concrete and the steel, respectively, $p_{n}^{+}$is the tensile strength. Variational principle then leads to the following definition: Find the minimum displacement vector $\boldsymbol{u}$ and the maximum traction vector $\boldsymbol{p}=\left\{p_{n}, p_{t}\right\}$ for the functional of entire energy $\mathrm{E}_{\text {ent }}$ :

$$
\begin{aligned}
\mathrm{E}_{\text {ent }}= & \frac{1}{2}\left(\int_{\Omega^{\prime}} \boldsymbol{\sigma}^{\mathrm{T}} \boldsymbol{\varepsilon} \mathrm{d} \Omega^{\prime}+\int_{\Omega^{\prime \prime}} \boldsymbol{\sigma}^{\mathrm{T}} \boldsymbol{\varepsilon} \mathrm{d} \Omega^{\prime \prime}\right)-\int_{\partial \Omega^{\prime \prime}} \boldsymbol{p}^{\mathrm{T}} \boldsymbol{u} \mathrm{d} \partial \Omega^{\prime \prime}+ \\
& +\int_{\Gamma}\left(\left|p_{t}\right|-C\right)\left|\left[u_{t}\right]\right| \mathrm{d} \Gamma+\int_{\Gamma}\left(p_{n}-p_{n}^{+}\right)\left[u_{n}\right] \mathrm{d} \Gamma
\end{aligned}
$$

where subscripts in denotation of domains mean $c=$ concrete, $s=$ steel, $\boldsymbol{\sigma}$ and $\boldsymbol{\varepsilon}$ are respectively the stresses and the strains in both bodies, $\Gamma$ is the interface, $\partial \Omega^{\prime \prime}$ is the external boundary of the representative element, $\mathrm{T}$ means transposition. It is usable to introduce the mixed formulation for the finite element method:

Let us write the total energy $J$ of both bodies assuming them separately:

$$
J(\boldsymbol{u}, \boldsymbol{p})=\Pi(\boldsymbol{u})-I(\boldsymbol{u}, \boldsymbol{p}),
$$

where

$$
\begin{gathered}
\Pi(\boldsymbol{u})=\frac{1}{2} a(\boldsymbol{u}, \boldsymbol{u})-\int_{\partial \Omega^{\prime \prime}}\left(\boldsymbol{p}^{\prime \prime}\right)^{\mathrm{T}} \boldsymbol{u}^{\prime \prime} \mathrm{d} \partial \Omega^{\prime \prime}, \\
I(\boldsymbol{p}, \boldsymbol{u})=\int_{\Gamma}\left(\left|p_{t}\right|-C\right)\left|\left[u_{t}\right]\right| \mathrm{d} \Gamma+\int_{\Gamma}\left(p_{n}-p_{n}^{+}\right)\left[u_{n}\right] \mathrm{d} \Gamma,
\end{gathered}
$$




$$
a(\boldsymbol{u}, \boldsymbol{u})=\int_{\Omega^{\prime}} \boldsymbol{\sigma}^{\mathrm{T}} \boldsymbol{\varepsilon} \mathrm{d} \Omega^{\prime}+\int_{\Omega^{\prime \prime}} \boldsymbol{\sigma}^{\mathrm{T}} \boldsymbol{\varepsilon} \mathrm{d} \Omega^{\prime \prime}
$$

The first stiffness matrix in (2) appears to be singular, while the second is regular, as the second matrix is created under assumption of periodic conditions. For the first matrix in order to be regularized has to be connected with the first by introducing some relation to the second matrix by connection of springs, for example. This trick enables us to connect both substructures (fibers and matrix) in a physically reasonable way.

From the above consideration it follows that the contact representation by spring stiffnesses $k_{\mathrm{n}}$ (normal direction) and $k_{\mathrm{t}}$ (tangential direction) can be characterized (normal and tangential directions are taken with respect to the surface of the fiber) and penalty like formulation can be considered in both cases: small deformation and large displacement theories. If the spring stiffness is high, the bond of fibers to matrix is defined, if some of contact conditions are violated, the stiffness lowers its value. The impact of this formulation ensures always the solution, if the fiber is not disconnected from the matrix in all nodal points. In this sense the interfacial relations can be written as:

$$
p_{n}=k_{n}\left[u_{n}\right] \quad p_{t}=k_{t}\left[u_{t}\right]
$$

and the interfacial energy $I$ in (8) is written as:

$$
I(\boldsymbol{p}, \boldsymbol{u})=\int_{\Gamma} k_{t}\left[u_{t}\right]^{2} \mathrm{~d} \Gamma+\int_{\Gamma} k_{n}\left[u_{n}\right]^{2} \mathrm{~d} \Gamma-\int_{\Gamma} C\left|\left[u_{t}\right]\right| \mathrm{d} \Gamma-\int_{\Gamma} p_{n}^{+}\left[u_{n}\right] \mathrm{d} \Gamma
$$

where spring stiffnesses $k$ play the role of penalties and the last terms in (10) represent the peak (strength) energies for both normal and tangential directions.
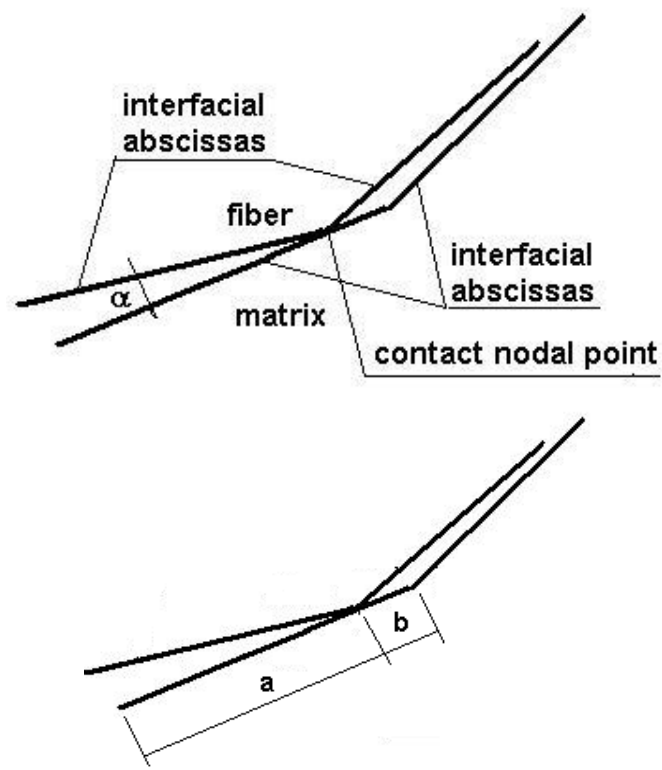

Figure 2: Calculation of forces after dissipation of interfacial energy. 


\section{Algorithm}

In the case of large displacements two possibilities have to be distinguished. The first consists in the situation which appears after disconnection of fibers from matrix. Then the interfacial energy disappears and if no new fiber-matrix contacts occur, the crack remains open. If the new touch of fibers with matrix would be found, see Fig. 2, new contact forces are generated according to "spring rule". The forces are divided into nodal points regularly, i.e., if the force is, say, $F$, then to the node near a we get $F \mathrm{~b} /(\mathrm{a}+\mathrm{b})$ and to the node near $\mathrm{b}$ the force $F \mathrm{a} /(\mathrm{a}+\mathrm{b})$ is transmitted. The similar formulation (7) takes place and the algorithm continues in the standard way.

If the angle $\alpha$ is equal to zero, the accumulated energy due to shear and normal forces does not disappeared and has to be taken into consideration. The new position of the nodal points has to be considered and additional energies are generated from the new constellation of the nodes. The energy functionals differ from the starting one, (7), by additional terms which are based again on "spring rule".

\section{Experiments with fibers}

In order to compare polypropylene fibers (PFRC) and modified polypropylene fibers (MPFRC) and steel fiber reinforced concrete (SFRC), four point bending tests have been carried out. In Fig. 3, results from four tests have been conducted

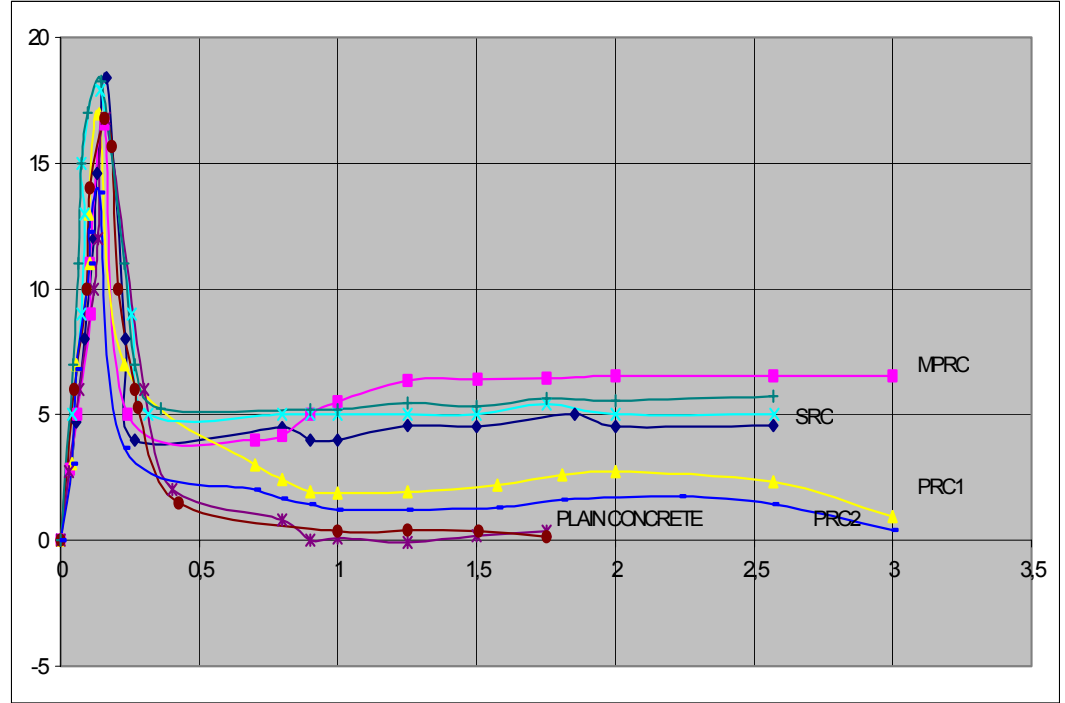

Figure 3: $\quad$ Comparison of results of all tests. 
on $400 \mathrm{~mm}$ spanned beam, $100 \times 100 \mathrm{~mm}^{2}$ was the cross-section. In the picture graph force and displacement is depicted. From Fig. 3 one can see the results from three tests with polypropylene fibers, one with steel fibers and one with modified polypropylene fiber. From the picture it is seen that there is much higher residual stress by MPRC and even local hardening in the region of softening appears. The hardening seems to be very steep. In the case of polypropylene fibers slight hardening is seen, too, but not that emphatic. In Fig. 4 a similar tests has been carried out for SFRC with obviously harder stiffness. The tests show that the results are much closed one to each other.

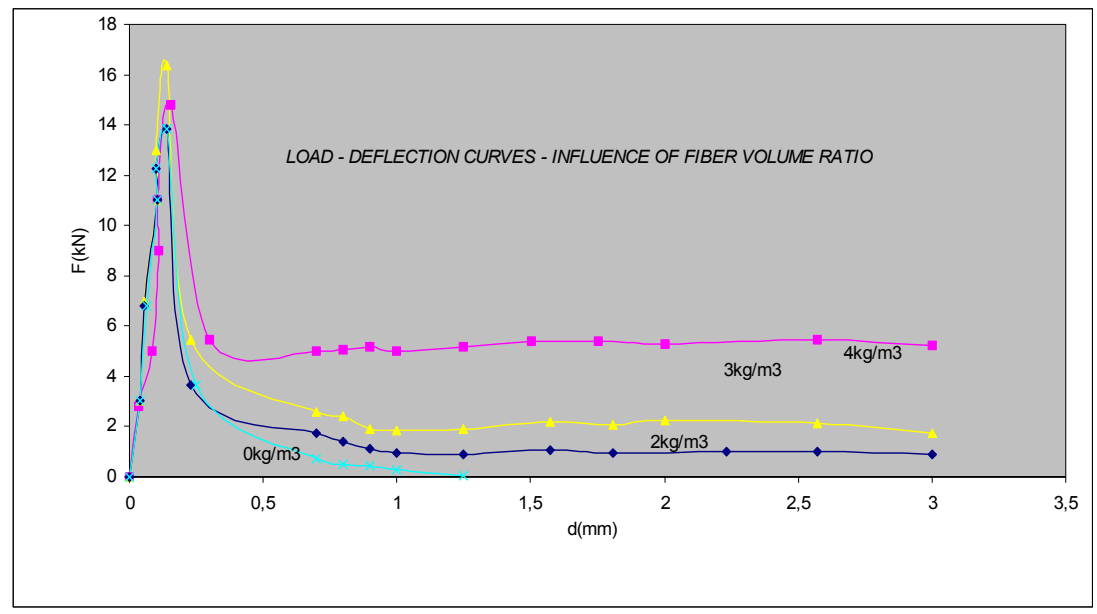

Figure 4: Influence of fiber volume ratio.

\section{Conclusions}

In this paper application of various fibers in large span structures is discussed. Particularly, envelopes in underground nuclear power stations are such where fiber reinforced concrete even has to be used for different reasons, which are mentioned in the above text. In case large span structures have to be the bearing element, pull of fibers out of concrete matrix cannot be simplified in calculation starting with small deformation theory. This is why large deformation theory is mentioned here and briefly described. Results from calculations are shown for various fibers with various shapes.

\section{Acknowledgements}

Financial support of GAČR, project number 103/08/1197 is appreciated. The research has also been supported by a grant of Ministry of Education of the Czech Republic number MSM6840770001. 